\title{
A Performance-Based Framework for Structural Resilience to Blast-Induced Damage
}

\author{
Shalva Marjanishvili, DSc, PE, SE and Brian Katz, PE \\ Hinman Consulting Engineers, Inc. \\ Spencer Quiel, PhD, PE \\ Lehigh University
}

\begin{abstract}
This paper proposes a framework for establishing quantitative measures and mathematically reproducible definitions of structural resiliency as it pertains to a structure's ability to minimize the potential for undesirable response to low-probability-high-consequence events. The resiliency assessment and design process follow a logical progression of steps starting with the characterization of hazards and continuing through analysis simulations, damage modeling, and loss assessment by balancing functional relationships between design tradeoffs and associated consequences. The outcomes of each subprocess are articulated through a series of generalized variables: topology, geometry, damage, and hazard intensity measures. A rigorous probabilistic framework permits consistent characterization of the inherent uncertainties throughout the process. The proposed framework is well suited to support the building design process through stochastic characterization of assessment measures. Using a stepwise approach, the framework facilitates a systemwide method to confront multihazard threat scenarios by establishing functional relationships between the development of appropriate models, design methods, damage acceptance criteria, and tools necessary for implementation. The proposed methodology can be implemented directly for assessment of project-specific performance criteria or can be used as a basis for establishing appropriate performance criteria and provisions to achieve resilient structural solutions at the outset of design.
\end{abstract}

\section{INTRODUCTION}

The recent past has witnessed unprecedented levels of structural failures in response to extreme loading events and structural deterioration. Despite engineers' efforts to implement an elevated level of design, these incidents have highlighted the significant threat to our built environment posed by low-probability-high-consequence events and the shortcomings of conventional design approaches in providing an effective engineering process to confront failure propagation as part of the design process. Emerging trends in the engineering community suggest the problem is rooted in the misnomer of achieving a "collapse proof" structure using design approaches that address a limited range of performance in response to specific extreme loading conditions to incrementally increase resistance. The resulting design, however, may be unable to cope with even slight variability in the initial design assumptions or structural response.

Given the need to preserve building functionality, designers are turning to concepts of resiliency which stress the need for a system to resist, adapt to, and recover from exposure to a broad range of hazards. The resiliency of a system is measured by its ability to mitigate the effects of an extreme load and minimize the recovery needed to restore functionality. The concept of resilience is applicable at multiple levels within the scale of the built environment, progressing from structural components to single structures to networks of structures to entire communities. Evaluations of resilience at each level are critical to the overall ability of our infrastructure to withstand and recover from extreme events. The framework developed in this paper will focus on the resilience of a single structure to blast loads and potential local damage. Frameworks at the structure level can then be used to provide input for resilience models at the network and community level.

This paper outlines a decision-based framework by which the magnitude of the consequences to blastinduced local damage can be calculated and used to assess structural resiliency. Current models define system resiliency as the time to full (or nearly full) recovery after a shock, insult, or disturbance (i.e., hazards). In this context, it is difficult to understand the engineering process by which to evaluate and implement resilience as part of the design process. This paper proposes an alternate definition of resilience in which the amount of required recovery is correlated with the amount and severity of damage inflicted by an air-blast threat. In this framework, the user determines the likely damage for a range of 
increasing threats and decides whether the corresponding consequences based on the sequence of damage (which quantifies the resilience) is acceptable. Current performance-based methodologies are reviewed and adapted to provide a procedural framework that is multideterministic and, therefore, more accessible to engineers who rely on the current state of practice. The mathematical formulation of the proposed approach is discussed, and a design example is provided in which the proposed framework is demonstrated.

\section{RESILIENCE VERSUS ROBUSTNESS}

The key issue confronted by collapse-resistant design approaches is the formulation of a building solution to resist an unexpected and unpredictable event without a priori knowledge of the location of the local damage and, thus, the building's load redistribution requirements. At the epicenter of progressive collapse theories and design practice is the concept of robustness. This quality of a structural system characterizes the extent to which stability can be maintained when equilibrium is perturbed. Collapseresistant design is essentially the practice of calculating structural robustness and enhancing the ability of the building to cope with extreme load conditions where it is necessary.

Lacking clear guidance in how to achieve robustness, engineers have extended their understanding of conventional structural theories in the pursuit of collapse-resistant design. The predominant technical methods employed in this exercise are deterministic in nature and rely on a series of assumptions to reconcile unknowns associated with damage scenarios, initial conditions that define system resistance, and observed variability in structural performance at the brink of collapse and provide an achievable path to implement collapse resistance. The design process is largely characterized by a component-by-component validation of the structure and subsequent local strengthening of the system until a prescribed level of robustness is achieved. This process is predicated on the assumption that robustness is a variable property of the structure, correlated to strength and load path redundancy. However, observed structural behavior in the aftermath of extreme loading events contradicts this assumption. There are many examples where seemingly highly redundant structures have failed and, conversely, where expected building failure was not observed.

This paper argues that the disparity between expected and observed building performance is rooted in the assumption that strength-based methods, applied at the component level effectively, will adequately alter the global resistance from which structural robustness is derived. Rather than consider the resistance as a sliding scale in relation to a fixed load, the proposed alternative is to consider robustness as a fixed property of the system that is uniquely tied to the structural configuration as expressed in Equation 1:

Robustness $=$ f(topology, geometry)

In this formulation of robustness, "topology" refers to the building shell configuration relative to the site. This property defines the expected exposure of the building to widespread or local concentrations of extreme loads. The "geometry" term refers to the layout of the structural load-bearing elements. Both are absolute properties that cannot be changed without modifications to the overall building configuration. In this way, once the building system's geometry has been defined, so too has the structure's robustness.

If robustness is held to be an absolute property of the system, then resilience represents the variable property that fluctuates with specific design decisions. Pursuit of resilience is typically considered to be an exercise in balancing the ability of a given structure to resist, adapt to, and recover from extreme events (see Equation 2). The "resistance" component of Equation 2 represents engineering effort to withstand a prescribed hazard. Load resistance allows the structure to achieve rapid recovery to a wide range of threats by avoiding damage. However, even robust structures may experience some damage when subject to design levels of extreme loads. To resist blast loads, building elements are designed to experience allowable levels of plastic deformation or cracking. Even if element "failure" (i.e., collapse) is avoided, damaged elements will require repair or replacement resulting in a temporary loss of building functionality. Resistance should therefore be provided, such that potential damage minimizes casualties and reduces the likelihood of catastrophic structural losses. The "adaptation" component is largely understood to consist of high-level emergency planning efforts to restore facility function in the aftermath of a catastrophe. The "recovery" component represents the process over time in which facility function is restored via repair and/or replacement. The perceived balance of these variables as it impacts system resilience is visually depicted in Figure 1 which plots functionality on a time scale.

Equation 3 revises the common expression of resilience to exclude the recovery and adaptation components, as these parameters cannot be easily quantified as part of engineering design efforts. The resistance component of resilience is broken into robustness and hazard parameters.

$$
\begin{aligned}
\text { Resilience } & =\mathrm{f}(\text { resistance }, \text { adaptation, } \text {, recovery }) \\
\text { Resilience } & =\mathrm{f}(\text { hazard }, \text { robustness })= \\
& =\mathrm{f}(\text { hazard }, \text { topology }, \text { geometry })
\end{aligned}
$$


In this modified expression of resilience, the structural performance associated with a specific system configuration is considered to be independent from the contribution of component strengthening to address a prescribed load or hazard. The resulting equation for resilience represents the specific hazard magnitude mitigated by a structural design with an assigned robustness. This definition of resilience allows engineers to quantify resilience and robustness in more certain terms and provides a basis to better assess post-event structural behavior. By extension, resilience, $\mathrm{R}$, can be expressed mathematically as the normalized integration of the functionality, $Q$, as shown in Figure 1 (Bocchini \& Frangopol, 2012), in which $Q$ is represented as a function of topology, T; geometry, G; and hazard, $\mathrm{H}$ :

$$
\mathrm{R}=\mathrm{f}(\mathrm{H}, \mathrm{T}, \mathrm{G})=\left(\frac{1}{\mathrm{t}_{\mathrm{r}}-t_{0}}\right)_{t_{0}}^{t_{r}} Q(H, T, G) d t
$$

\section{PROPOSED RESILIENCY FRAMEWORK}

The proposed framework is centered on a performance-based calculation of the consequences due to blast-induced local damage. The consequences, $\mathrm{C}$, quantify the loss of functionality which constitutes the initial condition needed for integration in Equation 4. As a starting point, a riskbased integration based on the total probability theorem is constructed, similar to that used by several stochastic performance-based approaches (Barbato, Petrini, Unnikrishnan \&, 2013; FEMA, 2012), to calculate the likely magnitude of the consequences based on the topology, geometry, and hazards:

$$
\begin{aligned}
& \lambda(c)=\iiint \int G(C \mid D M) \cdot p(D M \mid E R P) \times \\
& \times p(E R P \mid I M) \cdot p(I T \mid T) \cdot p(T) \times \\
& d D M \cdot d E R P \cdot d I M \cdot d T
\end{aligned}
$$

Where $G(\ldots \mid \ldots)$ is the conditional cumulative distribution function; $p(\ldots \mid \ldots)$ is the conditional probability density function; $T$ is the threat location

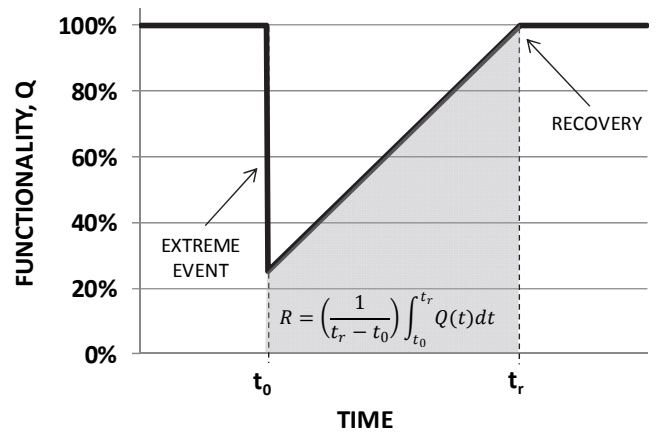

Figure 1. Graphical representation of functional recovery following an extreme event which causes damage and size (accounting for the standoff and orientation of the explosive threat to the structure as well as its charge weight); IM is the intensity measure (i.e., the magnitude of blast pressure and impulse due to the threat); ERP is the engineering response parameter (such as ductility, rotation, shear, or breach) due to the IMs and Ts; DM is the direct damage caused by the blast according to the ERPs; and $\mathrm{C}$ is the consequence of the damage (i.e., the resulting structural and/or functional losses). Equation 5 is solved by sequentially solving for the probability of exceedance for each successive parameter by integrating its probability density function as conditional of the upstream parameters. To achieve a quantitative solution, a conditional probability density function must, therefore, be developed, available, or assumed for each step of integration.

To reduce the number of required probability density functions, Equation 5 can be simplified by selectively designating some of the parameters as deterministic variables. The IM at every target on the structure can be assumed to be a deterministic function of the threat location and size, as is commonly done in blast engineering practice (DoD, 2008). If threats are examined one at a time, then the probability of DM becomes directly correlated to the IM and the integration over $\mathrm{T}$ is eliminated. Also, the integration over ERP can be eliminated if deterministic, predefined response limits are chosen (i.e., variability in the ERP is removed):

$$
\lambda(c)=\iint G(C \mid D M) \cdot p(D M \mid I M) \cdot d D M \cdot d I M
$$

Equation 6 is used to evaluate the consequences for each threat location which can then be compared either graphically or numerically for all likely or potential threat locations. Even though its integration has been simplified, Equation 6 still requires that the user provide probabilistic functions relating the damage to the blast effects as well as the consequences given the damage. To complete the transition toward using deterministic rather than probabilistic methods, we can replace the probabilistic functions $G(C \mid D M)$ and $p(D M \mid I M)$ with direct functions for $\mathrm{C}$ and $\mathrm{DM}$. As is done in practice, DM can be considered to be a deterministic function of the exceedance of the ERP by the corresponding IM. DM(IM) can be calculated using single degree of freedom analysis of the elements (DoD, 2008) according to response limits representing "failure" (USACE, 2006). The function for $C$ describes the increase of consequences as a function of the pattern of DM (i.e., as a function of DM given IM). $\mathrm{C}(\mathrm{DM} \mid \mathrm{IM})$ is a user-defined function that describes the increasing amount of structural loss (and therefore functional loss) associated with the location and extent of damage. The calculated value for total consequences can then be expressed as follows: 
$C(T)=\iint G(D M \mid I M) \cdot D M(I M) \cdot d D M \cdot d I M(7)$

The functions within the double integral in Equation 7 represent a surface that graphically describes the overall consequences in response to increase threat intensity for the given threat and geometry. The volume under this surface is calculated via the double integration and represents the initial condition for the calculation of resilience, $R$. If the shape of functionality $Q$ in Figure 1 and Equation 4 is assumed to include an instantaneous drop of magnitude, $\lambda(C)$, due to an extreme event at time, $t_{0}$, and is followed by a linear recovery to full functionality at time, $t_{r}$, then Equation 4 can be rewritten as follows:

$$
\mathrm{R}(\mathrm{T})=\left(\frac{1}{\mathrm{t}_{\mathrm{r}}-t_{0}}\right)_{t_{0}}^{t_{r}}\left[\left(\frac{C(T)}{\mathrm{t}_{\mathrm{r}}-t_{0}}\right) t+t_{0}\right] d t
$$

If the rate of recovery is assumed to be independent of the magnitude or type of functionality loss, the resilience can be more simply calculated as inversely proportional to the consequences that result from the intensity of the given threat:

$$
R(T) \approx \frac{1}{C(T)}
$$

The framework proposed in this paper establishes a correlation between the pattern and sequence of damage due to the spatial distribution of blast effects on the building envelope to the resulting consequences. In other words, the consequences in terms of structural loss are governed by the order and location of element failure as the intensity of the blast threat increases. By developing the consequences as a function of the damage due to the distribution of threat intensity (i.e., C(DM|IM)), the relative resilience of the building to damage caused by a range of threat intensities (i.e., DM(IM)) can be evaluated.

\section{EXAMPLE}

A simplified design example is provided to illustrate the implementation of the proposed resilient framework in the context of blast-resistant building design. Two levels of structural robustness are considered in which the frame is (1) not explicitly designed to resist local damage (i.e., conventional construction) and (2) designed to bridge over the loss of a single one-story column (i.e., current progressive collapse resistant design).

This example, ultimately, demonstrates the process by which resilience can be assessed and high-level design decisions can be made regarding structural system configuration as part of early stages of the design process where a fixed threat is considered.

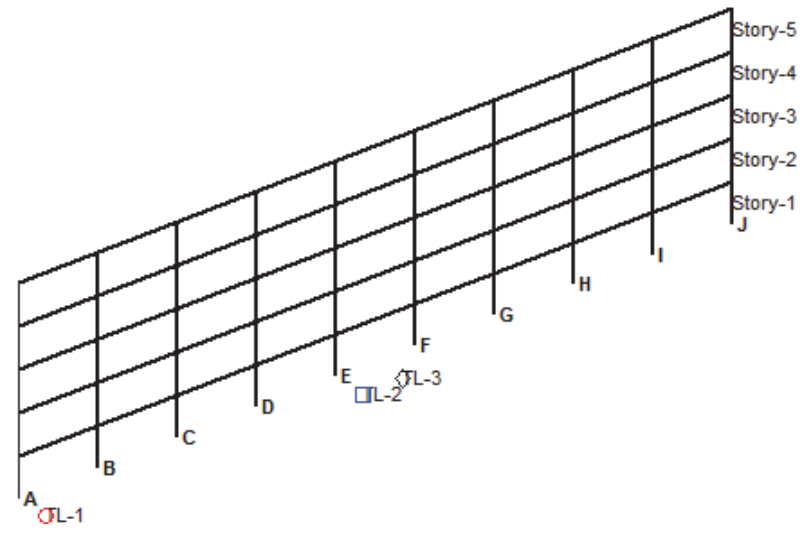

Figure 2. Example 2-D, 5-story frame

\subsection{Site Layout-Topology}

The prototype structure used to illustrate the proposed framework to calculate resilience is a twodimensional, 5-story frame, as shown in Figure 2. The column grid is numbered consecutively from left to right starting from Grid A to Grid J. Columns are spaced at 9 meters; story height is fixed at 4.5 meters. As indicated previously, all columns are assumed to be characterized by the same material and section properties such that the same blast load intensity will cause an exceedance of the selected ERP of any one column. In practice, variable element design would be accounted for in determining the sequence in which failure propagates from one column to the next given the IM distribution.

\subsection{Threat Locations and IM-Hazard}

For this example, three explosive weapon threats were considered. All threats are equal in magnitude and plan-dimension offset from the frame. The threats' locations are as followed and were selected to be consistent with current progressive collapse practice of targeting critical ground level columns:

- TL-1: Threat located at the end bay column A-1.

- TL-2: Threat located at the interior bay column $\mathrm{E}-1$, which is approximately middimension of the frame.

- TL-3: Threat is located at the center of the interior bay bound by columns $\mathrm{E}-1$ and $\mathrm{F}-1$ and represents the threat considered to result in potential simultaneous failure of two first floor columns.

For the purpose of illustrating the procedure of the proposed framework, we will first focus on TL-3. Figure 3 depicts a scatter plot of air-blast pressures and impulses (i.e., the blast intensity measure) for each column (e.g., E-1 is column line E at story 1 ) for TL-3. In Figure. 5, the points are ordered according to descending intensity as a percentage of the 
maximum intensity to identify columns that are exposed to the highest magnitude loads.

\subsection{Consequence and Damage-Geometry}

For Structure 2, the acceptable consequence would be the loss of functionality only at the two bays adjacent to the failure of a single one-story column (i.e., preventing collapse of the floors bridging over the failed column). The acceptable damage is therefore the single column failure. For Structure 1, the structure is not explicitly designed to withstand any damage and, therefore, no allowable consequences or damage has technically been chosen. For the purpose of this example, Structure 1 is used as a comparison of relative resilience to local damage to that of Structure 2.

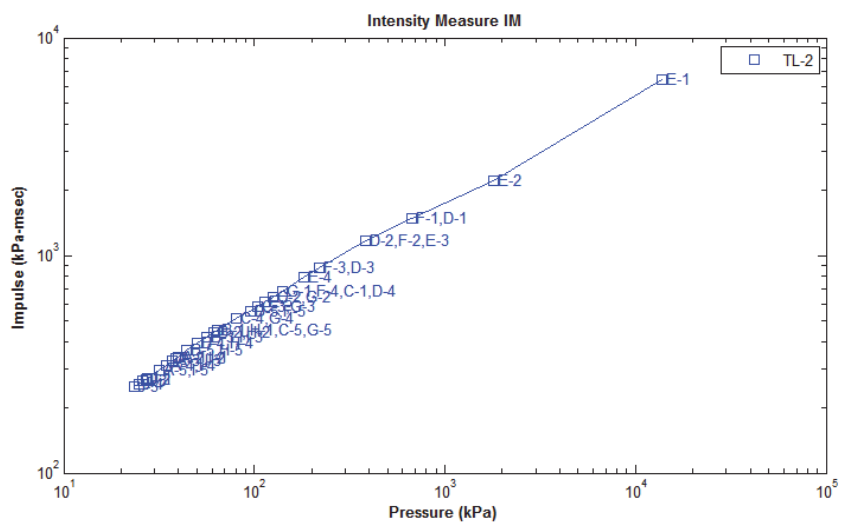

Figure 3. Hazard Intensity Measure (IM) scatter plot for each column for TL-3

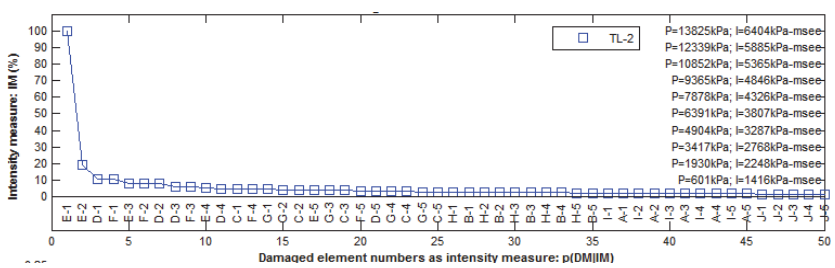

Figure 4. Topology plot of relative IM plot for all columns

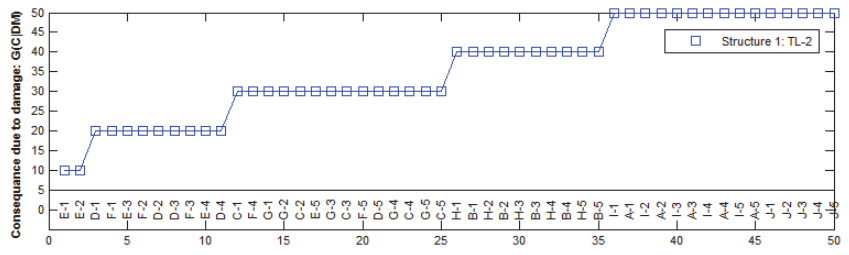

Figure 5. Geometry plot of consequence function for Structure 1, TL-3 (conventional design)

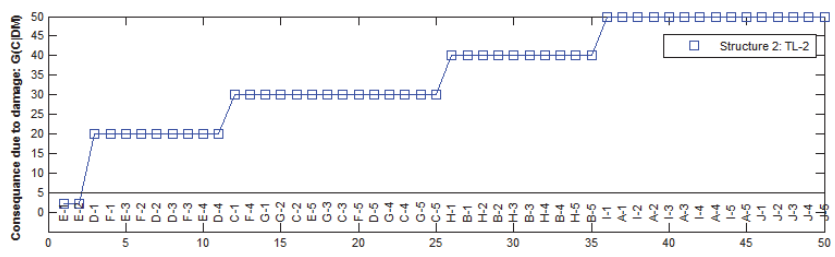

Figure 6. Geometry plot of consequence function for Structure 2, TL-3 (progressive collapse design)

\subsection{The Sequence of Damage}

Given the assumption that all columns are equal and bound by a singular ERP, columns with the greatest exposure to extreme loads are considered to also experience the greatest extent of damage. In this way, the example establishes a direct correlation between the DM and IM components of the mathematical formulation and simplifies the process of assessing damage propagation. As shown in Figure 4, for instance, column E-1 is expected to experience the highest loads and incur the greatest levels of damage. In practice, the IM field can be used to assess damage on a component-bycomponent basis to establish a design-specific correlation between the DM and IM parameters.

\subsection{Damage and Consequence Functions}

In general, the consequence function can be derived by rigorous finite element analysis of the structure to assess damage propagation given singular element failure. However, for the purpose of the illustration of the example, the consequence function is derived empirically based on the number of frame bays attributed to a specific column. In this way, failure of an individual column can be deterministically correlated to a structural loss measure. Each consequence function is unique not only to structure but also to threat location.

Using this method, the two structures of alternate robustness-design with and without progressive collapse resistance-can be compared in terms of consequence. Figure 5 illustrates the consequence function for the conventionally designed Structure 1, which does not include progressive collapse resistance. Failure of a typical interior column is assigned a consequence of ten bays lost-the two bays immediately adjacent to the column at ground level and the eight bays above. The formulation of the consequence function additionally considers the equal relationship of all columns. Therefore, the consequence of column E-5 failure encompasses a two-dimensional spread of failed columns below on Line $E$ as well as adjacent columns that are equidistant or closer to the threat location.

Figure 6 illustrates a similarly formulated consequence function for Structure 2, which does include collapse resistance at all column locations. Since this structure is able to bridge the loss of any single column, failure of one column is only expected to result in functional loss at the two adjacent bays. However, the propagation of damage that results from the failure of two adjacent columns at the same story (and beyond) produces the same consequence as for Structure 1 since the progressive collapseresistant design is not sufficient to address any larger extent of damage. 


\subsection{Consequence and Resilience}

An overall consequence measure (CM) can be obtained by multiplying the consequence function (C) of Figures 5 and 6 with the plot of relative IM portrayed in Figure 4, which is directly correlated to the Damage Measure (DM) for this example. Figures 7 and 8 show the CM surfaces for Structure 1 and Structure 2, respectively, for each considered threat location over the domain of IM and DM. The consequence $C(T)$ is then obtained by calculating the volume under each surface (i.e., by solving Equation 7).
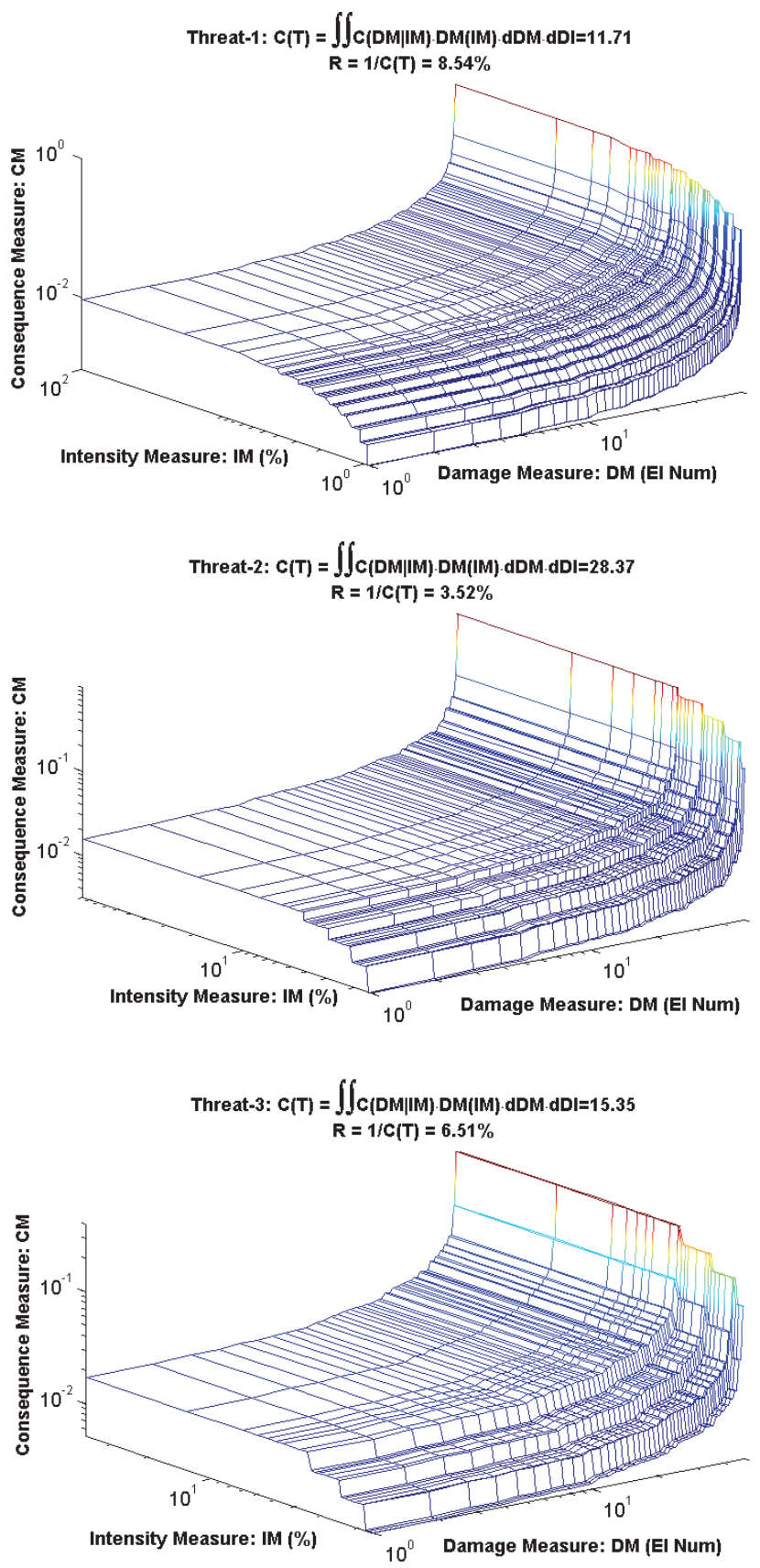

Figure 7. Consequence measure and resilience indicator for Structure 1
Additionally, Figures 7 and 8 indicate a Resilience Indicator (RI) for each structure and threat, which is a direct estimate of Equation 9. The consequence is a summation of the total consequence associated with the variable damage field across all elements. Thus, a high RI can be achieved through a combination of (1) consequence minimization for a given threat or (2) threat reduction for an expected consequence.
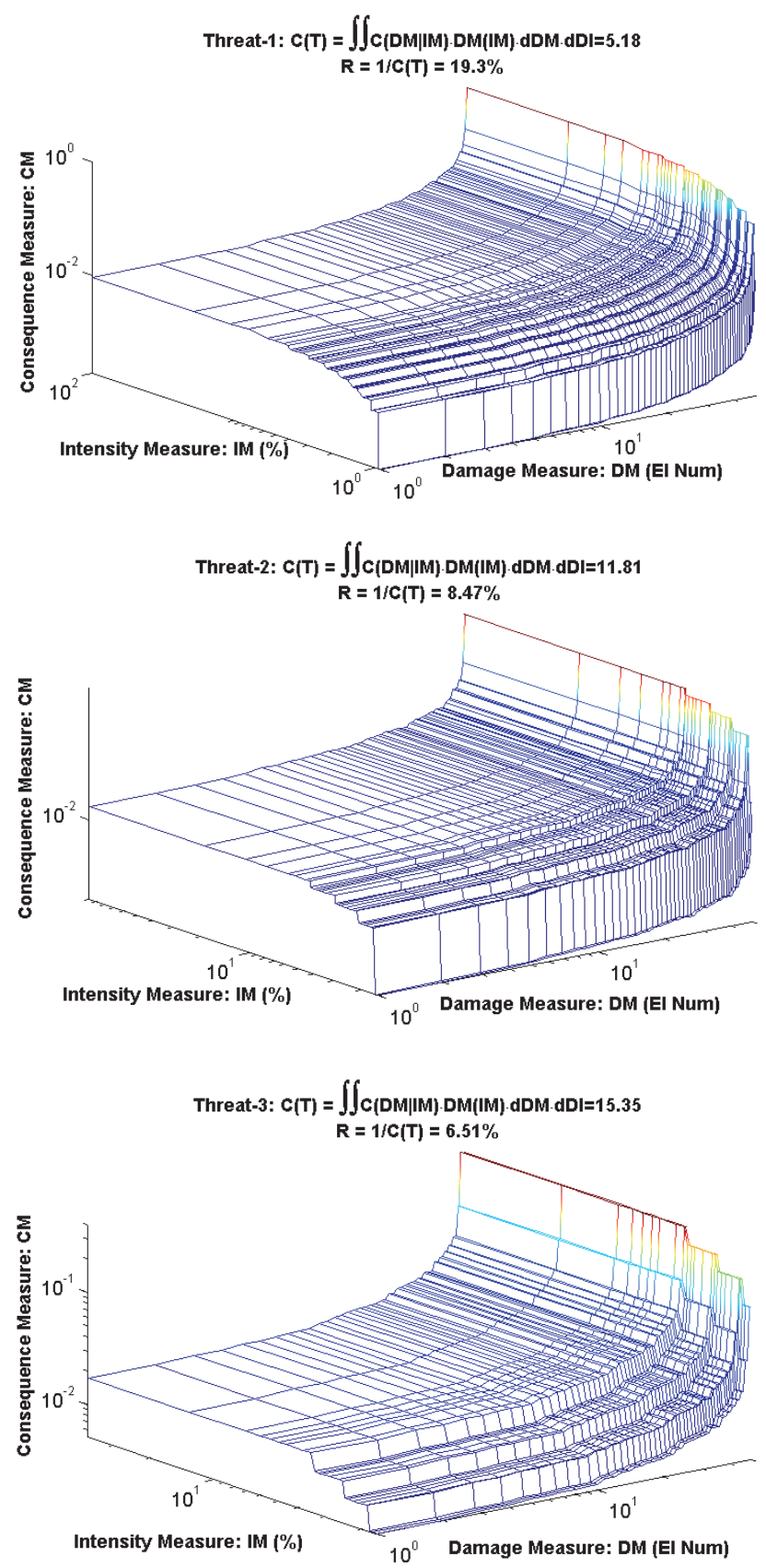

Figure 8. Consequence measure and resilience indicator for Structure 2 
Examining the results, several key conclusions can be made as follows:

- Structure 1 is most resilient to TL-1 $(\mathrm{RI}=$ $8.54 \%)$ and least resilient to $\mathrm{TL}-2$ (RI $=$ $3.52 \%)$.

- Structure 2 is similarly most resilient to TL-1 $(\mathrm{RI}=19.3 \%)$ but is least resilient to TL-3 (RI $=6.51 \%)$.

- Structure 2 is generally considered to be more resilient than Structure 1.

- The calculated RI for Structure 2 in response to TL-1 and TL-2 is roughly twice that of Structure 1 , providing a direct measurement of the benefit for the design to resist the loss of one element.

- Structure 1 and Structure 2 have the same RI for TL-3, which represents the threat scenario expected to cause simultaneous loss of two columns.

In general, the structure is only as resilient as the weakest link, or, in the case of this example, the RI associated with the limiting threat. Therefore, Structure 1 and Structure 2 are considered to have an RI of $3.52 \%$ (TL-2) and $6.51 \%$ (TL-3), respectively. Based on this assessment, if it is desired to elevate Structure 1 to the same level of resilience as Structure 2 without implementation of progressive collapse design, the design threat for Structure 1 needs to be increased by a factor of 1.9 (6.51/3.52). This will elevate the hazard component of the RI formulation to be more proportionate to the consequence for this example.

Based on these results, the structural engineer is able to make one of several decisions to dictate the final design of the structure:

1. "No Action"-Proceed with conventional design of the frame and accept consequence for the specified threats as represented in the results for Structure 1.

2. "Collapse Resistance"-Implement collapse resistance and accept the consequence for the specified threats as reflected in the results for Structure 2.

"Selective Strengthening"-Retain the configuration of the frame structure but selectively strengthen columns to minimize damage. This approach will not alter the formulation of the consequence function, which assumes failure, but results in an adjusted Consequence Measure (CM) and Resilience Indicator (RI).
3. "Reconfigure Building"-Alter the configuration of the frame. This approach will effectively change column orientation relative to the threat and result in a different Intensity Measure (IM) and Damage Measure (DM).

4. "Reconfigure Site"-Alter the configuration of the site. This action will require participation of the architect, site planners, and owners to change the site layout to mitigate the explosive threats. This action would involve the relocation of roadway, fences, locations of potential screening, or obstructions that may hinder surveillance of potential blast threat locations.

Each of these decisions can be framed in the context of overall impact to the building design, as well as construction cost, to determine the most ideal solution to optimize system resilience.

\section{CONCLUSIONS AND FUTURE WORK}

This paper outlines a decision-based framework by which the magnitude of the consequences to blastinduced local damage can be calculated and used to assess structural resiliency. The procedure for resiliency assessment starts with the characterization of hazards and calculates the resulting damage modeling and functional loss by deriving and subsequently balancing functional relationships between design and consequences. The outcomes of each process are articulated through a series of generalized variables, termed as topology, geometry, damage and hazard intensity measures. The framework is multideterministic and therefore more accessible to engineers who rely on the current state of practice. A design example using a 2-D structural frame was used to demonstrate the implementation of the framework.

The proposed framework has potential to be adapted for other hazard types such as impact or fire exposure that, like blast, are typically characterized by concentrated intensities. To address these additional hazards, future work is needed to develop methods to calculate the spatial distribution of hazard intensity and characterize the resulting damage and consequences. Adapting this procedure for impact loads would be fairly straightforward since most elements that are affected by the impact could be evaluated on a pass-fail basis similar to the use of performance criteria for blast load. Including fire exposure poses a greater challenge since elements with thermally induced material weakening and restraint of thermal expansion will develop a broader spectrum of damage across the structural system. 
Ideally, the framework would be extended to be multihazard to capture the resilience of the structure to the entirety of an extreme event (i.e., blast or impact followed by fire at the location of damage). In a previous study, the authors have examined the consequences of fire that follows an initial extreme event that results in local damage (Quiel \& Marjanishvili, 2012), and the incorporation of fire in the framework would allow users to leverage studies such as these to develop a holistic rather than hazard-dependent assessment of structural resilience.

\section{REFERENCES}

American Society of Civil Engineers. (2011). Blast protection of buildings (59-11). Reston, VA: American Society of Civil Engineers.

Baker, J. W., Schubert, M., Faber, M. H. (2008). On the assessment of robustness. Structural Safety, 30(3), 253-267. http://dx.doi.org/10.1016/ j.strusafe.2006.11.004

Barbato, M., Petrini, F., Unnikrishnan, V. U., \& Ciampoli, M. (2013). Performance-based hurricane engineering (PBHE) framework. Structural Safety, 45, 24-35. http://dx.doi.org/10.1016/j.strusafe. 2013.07.002

Bocchini, P., \& Frangopol, D. M. (2012). Optimal resilience- and cost-based postdisaster intervention prioritization for bridges along a highway segment. Journal of Bridge Engineering, 17(1), 117-129. http://dx.doi.org/10.1061/ (ASCE)BE.1943-5592.0000201

Department of Defense. (2008, December 5). Structures to resist the effects of accidental explosions (UFC 3-340-02). Washington, D.C.: U.S. Department of Defense. Retrieved from http://www.wbdg.org/ccb/browse_cat.php?c=4

Department of Defense. (2013). Design of buildings to resist progressive collapse (UFC 4-023-03). Washington, D.C.: U.S. Department of Defense. Retrieved from http://www.wbdg.org/ccb/ browse_doc.php?d=4430

Federal Emergency Management Agency. (2012). Seismic performance assessment of buildings (FEMA P-58). Washington, D.C.: Federal Emergency Management Agency. Retrieved from https://www.fema.gov/media-library/assets/ documents $/ 90380$
Marjanishvili, S., Katz, B., \& Hinman, E. (2012). Current analysis methods and structural collapse mitigation. In A. M. Dolhon, M. J. Drerup, A. D. de Len, J. B. Kardon, D.S. Hancock, \& S. -e. Chen (Eds.) Forensic Engineering 2012 (pp. 881-890). San Francisco, CA: American Society of Civil Engineers. http://dx.doi.org/10.1061/ 9780784412640.093

MathWorks. (2013). MATLAB [computer software]. Natick, MA: MathWorks.

McAllister, T. (2013). Developing guidelines and standards for disaster resilience of the built environment: A research needs assessment (NISTTN-1795). Gaithersburg, MD: National Institute of Standards and Technology. http://dx.doi.org/ 10.6028/NIST.TN.1795

Nafday, A. M. (2011). Consequence-based structural design approach for black swan events. Structural Safety, 33(1), 108-114. http://dx.doi.org/10.1016/ j.strusafe.2010.09.003

Quiel, S. E., \& Marjanishvili, S. M. (2012). Fire resistance of a damaged steel building frame that has been designed to resist progressive collapse. Journal of Performance Constructed Facilities, 26(4), 402-409. http://dx.doi.org/10.1061/ (ASCE)CF.1943-5509.0000248

Technical Support Working Group. (2004). Vehicle borne improvised explosive devices in worldwide terrorism: A contemporary open source analysis. Washington, D.C.: U.S. Department of Defense.

U.S. Army Corps of Engineers. (2008). Single degree of freedom structural response limits for antiterrorism design (PDC-TR 06-08). Vicksburg, MS: U.S. Army Corps of Engineers. Retrieved from https://pdc.usace.army.mil/library/tr/06-08

U.S. General Services Administration. (2003). Progressive collapse analysis and design guidelines for new federal office buildings and major modernization projects [PDF]. Washington, D.C.: U.S. General Services Administration. Retrieved from http://www.ara.com/Projects/ docs/PCADG.pdf

Whittaker, A., Hamburger, R., \& Mahoney, M. (2003). Performance-based engineering of buildings for extreme events. In AISC-SINY symposium on resisting blast and progressive collapse. New York, NY: American Institute of Steel Construction. 